\title{
Successful Closure of a Ventricular Septal Defect Using a Novel Non-Prosthesis Touching Technique Following Mechanical Aortic Valve Replacement
}

\author{
Xinghua Gu ${ }^{\mathrm{a}}$, Hourong Sun ${ }^{\mathrm{a}}$, Lei Zhang ${ }^{\mathrm{b}}$, Changjing Qi ${ }^{\mathrm{c}}$, Kejie Caic \\ Xiquan Zhang, d
}

\begin{abstract}
Percutaneous intervention is preferred over reoperation for the treatment of iatrogenic membranous ventricular septal defects (VSDs). During the standard percutaneous procedure, an arterio-venous loop is used for occluder deployment, entailing the risk of device impingement on the prosthetic aortic valve, which may cause serious complications or even death. In this report, we describe a novel non-prosthesis touching procedure for the closure of a VSD in a patient with prior aortic valve replacement. The unique feature of this technique is the use of an apex-venous loop for occluder deployment, which prevents the device impingement on the prosthetic valve, thus avoiding difficulties, lengthy operation and serious complications associated with the standard procedure. Immediate and 1-year follow-up results showed that the VSD was successfully closed and no serious complications were observed.
\end{abstract}

Keywords: Iatrogenic ventricular septal defect; Transcatheter closure; Aortic valves; Arterio-venous loop; Apex-venous loop

\section{Introduction}

Iatrogenic membranous ventricular septal defects (VSDs), most commonly seen after aortic valve replacements, are rare complications. Percutaneous transcatheter closure of these iatrogenic VSDs has increasingly become the preferred choice of therapy [1]. Since Holzer and colleagues reported two cases of successful percutaneous retro-aortic closure of iatrogenic VSD in 2004 [2], a few more similar case reports have been

\footnotetext{
Manuscript accepted for publication October 05, 2016

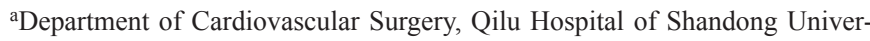
sity, Jinan, China

bepartment of Cardiology, Qilu Hospital of Shandong University, Jinan, China

${ }^{\mathrm{c} H y b r i d ~ O p e r a t i n g ~ R o o m, ~ Q i l u ~ H o s p i t a l ~ o f ~ S h a n d o n g ~ U n i v e r s i t y, ~ J i n a n, ~ C h i n a ~}$ ${ }^{\mathrm{d} C}$ Corresponding Author: Xiquan Zhang, Department of Cardiovascular Surgery, 107 Wenhua West Road, Jinan 250012, China.

Email: drzhangxiquan@sina.com
}

doi: http://dx.doi.org/10.14740/jocmr2777w published [1, 3-5]. An arterio-venous loop has been used for occluder deployment during the standard percutaneous procedure, entailing the risk of device impingement on the prosthetic aortic valve, which may cause serious complications or even death [1-6]. In this report, we described a novel noprosthesis-touching technique using an apex-venous loop for successful closure of a VSD in a patient following aortic valve replacement.

\section{Case Report}

In February 2015, a 47-year-old man with prior aortic valve replacement was admitted to the Department of Cardiovascular Surgery, Qilu Hospital after experiencing palpitation with exertion. Transthoracic echocardiography revealed a $5 \mathrm{~mm}$ membranous VSD close to the inferior suture of the prosthetic valve. Interventricular septum and anterior left ventricular wall were thickened. The function of the prosthetic valve was normal. Considering that the patient had already had an open heart surgery and that the percutaneous procedure has the risk of device impingement on aortic valve, we designed and performed a non-prosthesis touching procedure to close the VSD. Written informed consent was obtained from the patient, and the research was approved by the Research Ethics Committee, Qilu Hospital of Shandong University.

The procedure was completed in our hybrid operating room. Under general anesthesia, an $8 \mathrm{~cm}$ left anterolateral thoracotomy was performed in the fifth intercostal space. After the cardiac apex was well exposed, two apical purse string sutures (Prolene 2-0, large needle with two interrupted Teflon pledgets; Ethicon Inc, Somerville, NJ) in the bare spot of the anterior wall were placed deeply in the myocardium, close to the apex and lateral to the left anterior descending coronary artery. A 5-F sheath (Cordis Corporation, Miami Lakes, FL) was positioned at the apex and a 5-F pigtail (Cordis Corporation) was advanced into the left ventricular (LV) cavity by antegrade approach. Angiography in $40^{\circ}$ left anterior oblique view with $20^{\circ}$ cranial angulation confirmed the VSD (Fig. 1a) that was close to the inferior suture of the prosthetic valve, with a diameter of about 8 and $4 \mathrm{~mm}$ at left and right ventricular side, respectively. Pigtail catheter angiography of aortic root showed no aortic paravalvular leakage or regurgitation. A 5-F and a 6-F sheath (Cordis Corporation) were positioned in 

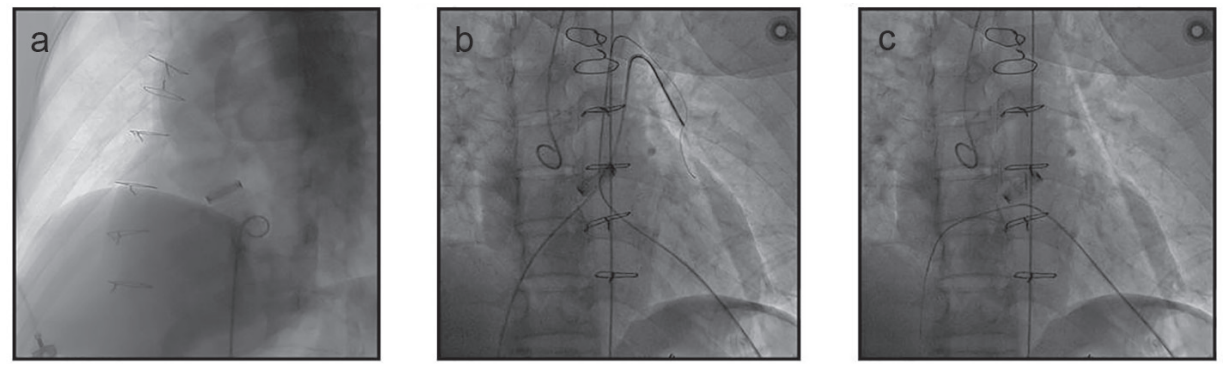

Figure 1. Angiography assessment and the establishment of the apex-venous loop. (a) LV angiography confirmed the membranous VSD. (b, c) Through the apex, a guide wire was advanced across the VSD to the right ventricle and then to the left pulmonary artery where it was snared (b) and exteriorized from the RFV (c), thus the apex-venous loop was established.

the right femoral artery (RFA) and right femoral vein (RFV), respectively. A $260 \mathrm{~cm} 0.035 "$ guide wire (Terumo Corporation, Tokyo, Japan) was advanced through the apex, across the VSD to the right ventricle and then to the left pulmonary artery where it was snared (Fig. 1b) and exteriorized from the RFV (Fig. 1c), thus an apex-venous loop was established.

The loop wire-assisted technique was then used for occluder deployment. From the RFV over the loop wire, a 7-F delivery sheath was advanced through the VSD into LV cavity and an 8-mm membranous VSD occluder (Lifetech Ltd, Shenzhen, China) was delivered. Occluder deployment was performed with the distal tip of the delivery sheath pointing to the apex (Fig. 2a). The device was pulled towards VSD until the left disc reached beneath the valve edge, and then the left disc was carefully opened and positioned followed by the opening of waist and the right side of the occluder (Fig. 2b). Repeated angiography of LV confirmed that the occluder was positioned properly, there was no residual shunt, and opening and closing of the valve leaflet was normal. The loop wire was then removed and the occluder was released (Fig. 2c). The apex was securely closed using the previously placed two purse string sutures. A soft chest drain tube was inserted, and the chest wall and incision were closed in a routine fashion.

The operation and fluoroscopic time were 52 and $16 \mathrm{~min}$, respectively. The hemodynamic indices were stable during the entire procedure. The patient was extubated at $2 \mathrm{~h}$ and discharged at 7 days after the intervention. At 1-, 3-, and 6- and 12-month follow-ups, the function of the prosthetic valve was normal. There was no atrioventricular block, hemolysis, resid- ual shunt, device embolization, device dislocation, infective endocarditis, or new-onset aortic/tricuspid regurgitation.

\section{Discussion}

A significant concern for the use of an arterio-venous loop in transcatheter closure of iatrogenic VSDs is the interference with valve function by placement of the device [1-5]. Drop of systemic blood pressure [1, 3-5], and transient significant aortic regurgitation were observed when the device was across the leaflet of the aortic valve [4]. To maintain a stable blood pressure, vasopressors were used [4]. Additionally, difficulties in positioning the device were encountered $[1,4]$, and lengthy operation was also experienced [4]. Use of the apex-venous loop in this study prevented device impingement on the prosthetic valve, thus avoiding the difficulties, lengthy operation, and serious complications associated with the standard procedure.

\section{Conflicts of Interest}

Authors have no conflicts of interest to declare.

\section{Grant Support}

Dr. Xinghua Gu's research was supported by the Independent Innovation Foundation of Shandong University (grant no.
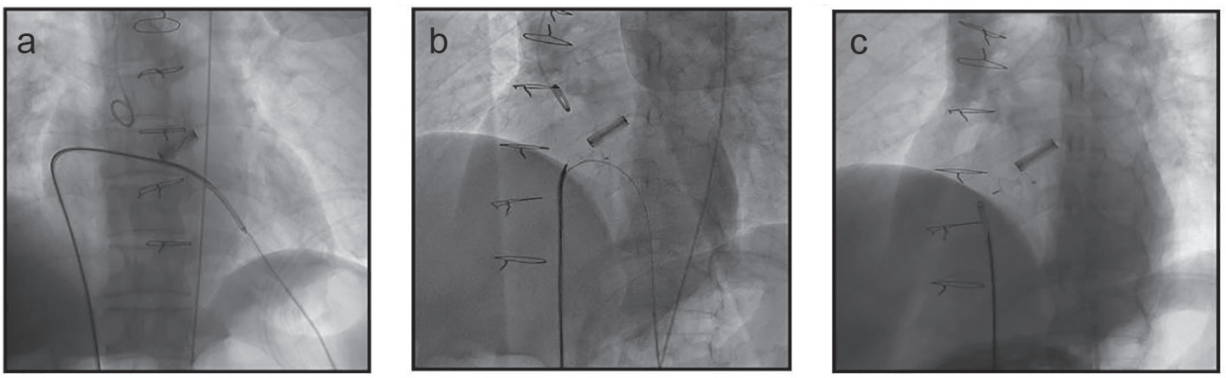

Figure 2. Occluder deployment through the apex-venous loop. (a) Occluder deployment was performed with the distal tip of the delivery sheath pointing to the apex. (b) The left disk was carefully opened and positioned beneath the edge of mechanical valve followed by the opening of the waist and the right side of the occluder. (c) The occluder was released and the loop wire was withdrawn after final LV angiography confirmation. 
2012TS171) and the Research Fund for Outstanding Young Scientists of Shandong Province (grant no. 2006BS03014), China.

\section{References}

1. Levi DS, Salem M, Suh W. Transcatheter closure of an iatrogenic ventricular septal defect after SAPIEN valve implantation. Catheter Cardiovasc Interv. 2015;85(3):E9094.

2. Holzer R, Latson L, Hijazi ZM. Device closure of iatrogenic membranous ventricular septal defects after prosthetic aortic valve replacement using the Amplatzer membranous ventricular septal defect occluder. Catheter Cardiovasc Interv. 2004;62(2):276-280.
3. Dodos F, Fehske W, Hoppe U, Sreeram N. Transcatheter closure of iatrogenic perimembranous ventricular septal defect following prosthetic aortic valve replacement. Clin Res Cardiol. 2008;97(1):53-55.

4. Noble S, Ibrahim R. Transcatheter membranous ventricular septal defect closure through a mechanical aortic prosthesis using the Amplatzer membranous ventricular septal defect occluder. Catheter Cardiovasc Interv. 2009;73(2):167-172.

5. Matsumoto T, Yeow WL, Kar S. Percutaneous closure of iatrogenic ventricular septal defect following surgical aortic valve replacement using two different approaches. J Invasive Cardiol. 2013;25(2):E42-44.

6. Horstkotte D, Jehle J, Loogen F. Death due to transprosthetic catheterization of a Bjork-Shiley prosthesis in the aortic position. Am J Cardiol. 1986;58:508-515. 